\title{
Thrombolysis Using Liposomal-Encapsulated Streptokinase: An In Vitro Study
}

P. D. Nguyen, E. A. O'Rear, A. E. Johnson, R. Lu, B. M. Fung

First Published December 1, 1989 Research Article

Article Information

Proceedings of the Society for Experimental Biology and Medicine, Volume: 192 issue: 3, page(s): 261-269

Issue published: December 1, 1989

\section{Abstract}

The clot-lysing ability of streptokinase (SK) was examined using membrane-bound thrombi. Encapsulation of SK in large unilamellar phospholipid vesicles (liposomes) resulted in entrapping approximately $30 \%$ of its original activity. Measurements of streptokinase activity for liposomal-encapsulated streptokinase (LESK) indicated little loss of activity or leakage in Tris-buffered saline over a 24-hr period at temperatures of 4 and $23^{\circ} \mathrm{C}$. However, incubation of free SK and LESK in platelet-poor plasma (PPP) at $37^{\circ} \mathrm{C}$ resulted in a decrease of SK activity. The retention of SK activity in LESK was considerably higher than that of unentrapped SK. Clot-dissolving time (CDT) was measured by monitoring the pressure drop during slow filtration in plasma through membrane-bound thrombi. The results indicated that both LESK and free SK were able to activate the fibrinolytic system. Without prior incubation in PPP at $37^{\circ} \mathrm{C}$, the CDT of a SK and PPP mixture (SK/PPP) was $10.7 \pm 1.9 \mathrm{~min}(n=12)$, while that of a LESK and PPP mixture (LESK/PPP) was $12.4 \pm 1.7 \mathrm{~min}(n=12)$. The CDTdetected clot-lysing abilities of both SK and LESK were diminished by incubation in PPP, but to different extents. After 15- and 30-min incubations, the CDT of SK/PPP increased significantly to $15.5 \pm 1.5$ and $24.1 \pm 2.4 \min (n=5, P<0.05)$, respectively. In contrast, the CDT of LESK/PPP increased to $13.3 \pm 0.8 \mathrm{~min}(n=5)$ after $15 \mathrm{~min}$ of incubation and to $16.0 \pm 1.1 \mathrm{~min}(n=5, P<0.05)$ after a 30-min incubation. These results suggest that entrapment of SK in liposomes preserves the thrombolytic potential of the plasminogen activator by limiting its exposure to the components of the plasma. 